\title{
Catálogos de Tesis de Posgrado: Ciclos 2004-2007 y 2008-2009 Sistematización Documental de Producción de Tesis de Posgrado en Diseño Proyecto de Investigación $\mathrm{N}^{\circ} 16.1$ Equipo de Investigación ${ }^{(1)}$ \\ Fabiola Knop por Facultad de Diseño y Comunicación, Universidad de Palermo (ARG)
}

\begin{abstract}
Resumen: El Proyecto de Investigación 16.1 presenta y pone en evidencia -a partir de los trabajos de tesis Ciclos 2004-2207 y 2008-2009- los lazos que se fueron tejiendo entre la formación académica con sus procesos, criterios y mecanismos absolutamente heterogéneos, y la destreza propia de cada disciplina, pudiendo validar a lo largo del camino un modelo teórico de competencias interculturales transversales propias de cada tesis. La articulación de todos los proyectos de Tesis, dan cuenta de la extensa y calificada labor científica propuesta, en cada uno de los niveles de investigación que trabajan en el resultado final del Proyecto, propone una búsqueda intencionada de conocimientos y soluciones a problemas que pueden ser de carácter cultural y/o formativo disciplinar. Finalmente da a conocer las categorías teóricas propuestas en cada trabajo, aspectos que los hace originales y hasta algunos plausibles de ser publicados académicamente.
\end{abstract}

Palabras clave: Conocimiento - Cultura - Diseño - Educación - Investigación - Metodología - Proyectos - Tesis

[Resúmenes en inglés y portugués en la página 203]

${ }^{(1)}$ Los CVs del Equipo de Investigación pueden consultarse en el Capitulo Directores de Líneas y Coordinadores de Proyectos de esta misma Edición.

\section{Acerca del Proyecto 16.1}

Catálogos de Tesis de Posgrado: Ciclos 2004-2007 y 2008-2009

Sistematización Documental de Producción de Tesis de Posgrado en Diseño

El Proyecto 16.1 Catálogos de Tesis de Posgrado, Ciclos 2004-2007 y 2008-2009: Sistematización Documental de Producción de Tesis de Posgrado en Diseño, reúne y sistematiza los resultados de las investigaciones realizadas por estudiantes del posgrado en 
Diseño. Se disponen de manera accesible, gráfica y digital, para ser utilizados como antecedente, medio de consulta y fuente de información para toda la comunidad académica y científica.

Avanza en una organización, sistematización, fundamentación y articulación del ámbito de la investigación científica y del tejido de reflexiones que emanan de las investigaciones realizadas en el Posgrado en Diseño de la Universidad de Palermo con la coordinación de Fabiola Knop (UP), con el fin de generar un corpus sólido de los cruces de pensamientos, saberes y teorías, riqueza y multiplicidad de perspectivas de abordaje sobre las áreas del diseño y la comunicación, que construyen un reservorio gráfico y digital, el que compone un espacio colaborativo, y un instrumento de difusión y transferencia a la comunidad académica regional e internacional.

Las reflexiones y los resultados obtenidos en el Proyecto 16.1 Catálogos de Tesis de Posgrado, Ciclos 2004-2007 y 2008-2009 se continúan desarrollando en el Proyecto 16.2 Catálogo de Tesis de Posgrado $3^{\circ}$ Edición: Ciclo 2010-2011, siendo que pertenece a una Línea vinculada estructuralmente a la Producción de las Tesis de los Posgrados en Diseño y al Programa Estímulo de la Facultad, por lo tanto existe una continuidad natural entre el desarrollo de las tesis y las investigaciones, su sistematización periódica en los Catálogos respectivos en el Reservorio gráfico y digital. Los Posgrados en Diseño, el Programa Estímulo y esta Línea avanzan de manera simultánea e integrada desde su creación, en el marco de la misma Línea de Investigación y bajo la misma Directora Fabiola Knop.

Sus principales objetivos son:

- Recopilar, organizar y catalogar los documentos de Tesis de Posgrado en Diseño y del Programa Estímulo a la Investigación, para promover la circulación accesible de conocimientos específicos del campo, e intervenir en las estrategias de regulación de las líneas temáticas y la calidad de las producciones.

- Sistematizar la producción de tesis e investigaciones para facilitar su accesibilidad

- Generar disponibilidad de estos contenidos para su uso como fuente de la información

- Establecer diagnósticos de la producción del conocimiento para regular posibles intervenciones curriculares y de gestión.

\section{Acerca de la Línea 16}

Reservorio Gráfico y Digital de Tesis de Posgrado en Diseño. Sistematización Documental de Producción de Tesis de Posgrado e Investigaciones en Diseño dirigida por Fabiola Knop se desarrolla de manera ininterrumpida desde 2001 en la Facultad de Diseño y Comunicación (UP, Argentina), e incluye hasta el momento siete proyectos finalizados, los que incluyen las tesis del Posgrado en Diseño: el 16.1. Catálogos $1^{\circ} \mathrm{y} 2^{\circ}$ Edición Ciclos 2004-2007 y 2008-2009, 16.2. Catálogo 3Edición Ciclo 2010-2011, 16.4 Catálogo 4Edición Ciclo 2012-2013, 16.5 Catálogo 5Edición Ciclo 2014-2015, y 16.6 Catálogo 6Edición Ciclo 2016-2017 coordinados por Fabiola Knop (UP), y los que pertenecen al Programa Estímulo a la Investigación: 16.3. Catálogo de Investigación $1^{\circ}$ Edición 2007- 
2015 coordinado por Marina Matarrese (UP), y 16.7. Catálogo de Investigación $2^{\circ}$ Edición 2016-2019, coordinado por Mercedes Pombo (UP).

\section{Mapa de Áreas y Proyectos}

El Proyecto 16.1 se vincula está vinculado con todas las Carreras de la Facultad de Diseño y Comunicación puesto que los lineamientos abordados son transversales a los contenidos relacionados con el Diseño en sus múltiples manifestaciones. Asimismo, sus resultados tienen una capacidad de impacto en los proyectos de Graduación de las Carreras de Grado de la Facultad, en las Tesis de Posgrado en Diseño, en las Líneas del Programa de Investigación en Diseño y en la Comunidad Académico Científica asociada, a través de las diferentes redes locales e internacionales creadas de la Facultad de Diseño y Comunicación.

\section{Productos y Resultados}

\section{a)- Publicaciones}

Cuaderno del Centro de Estudios de Diseño y Comunicación No38. (2011) Maestría en Gestión del Diseño y Maestría en Diseño de la Universidad de Palermo [Catálogo de Tesis. 2Edición. Ciclo 2008-2009]. Coordinación Fabiola Knop. Año XVIII, Diciembre 2011, Buenos Aires, Argentina. ISSN: 1668-0227. Esta publicación documenta y comunica los resultados alcanzados en el proyecto de investigación 16.1. Catálogos $1^{\circ} y 2^{\circ}$ Edición Ciclo 2004-2007 y Ciclo 2008-2009 de la Maestría en Diseño de la Universidad de Palermo, y a continuación se detallan los autores y artículos contenidos en ella:

Egresados de la Maestría en Diseño de la Universidad de Palermo (Pp. 11 a 17)

Álvarez Saavedra Eugenia Beatriz El cuero como material constructor de la identidad en Argentina (Pp. 19 a 20)

Bruzzone Virginia Soledad Barreras comunicacionales en las innovaciones tecnológicas dentro del sector agropecuario argentino (Pp. 21 a 24)

Gomez Barrera Yaffa Nahir Ivette (2009) La cultura del diseño, estrategia para la generación de valor e innovación en la Pyme del Área Metropolitana del Centro Occidente, Colombia (Pp. 25 a 27)

Paz y Miño Belén (2009) Los elementos distintivos de la Chola Cuencana, como material significante de un nuevo discurso proyectual (Pp. 28 a 29)

Castellanos Alvarado Sandra (2009) Modelo de interacción para transferencia de diseño a comunidades productivas emergentes (Pp. 29 a 30)

León Rincón Mauricio (2009) El relato de ciencia ficción como herramienta para el diseño industrial (Pp. 30 a 33)

Mussuto Gabriela (2009) Diseño no es moda, y moda no es diseño de indumentaria (Pp. 34 a 34)

Sánchez Juanita (2009) Reflexiones sobre la desvinculación del vestuario cinematográfico de su contexto y su resignificación como objeto plástico (Pp. 34 a 37) 
Bedoya Mosquera David (2009) El diseño como factor de optimización del consumo de productos alimenticios (Pp. 37 a 37)

González Aspera Alma (2009) Realidad virtual 3D como instrumento de comunicación multisensorial no inmersivo en instrumentos pedagógicos (Pp. 37 a 42)

Correa Cifuentes Oscar Humberto (2009) Acuñación y definición del término: Diseño Propagandístico (Pp. 42 a 45)

Valero Ramírez Diana (2009) Recobrando el Patrimonio vivo de Ciudad Perdida implementando el diseño en un circuito turístico cultural (Pp. 46 a 48)

Calle Molina Carlos Sebastián (2009) La marca ciudad de Cuenca como un elemento de participación social (Pp. 48 a 51)

Ponce Elena (2009) Espacio itinerante para la difusión de la mesa de fritos (Pp. 51 a 51 )

Vélez Jaramillo Paulina Los circuitos culturales en la construcción de marca ciudad. Caso Medellín (Pp. 52 a 53)

Camargo Silva Alex (2009) El Graffiti: una manifestación urbana que se legitima (Pp. 53 a 57)

Bedoya Mosquera David (2009) El diseño como factor de optimización del consumo de productos alimenticios (Pp. 57 a 57)

Ladino Velasquez Ariel (2009) El pensamiento complejo como herramienta para nuevas propuestas de diseño en objetos de uso (Pp. 57 a 61)

Alvarez Rojas Isabel Cristina (2009) Empaques y consumo de medicamentos: factores de cambio en el diseño y en la calidad de vida de los adultos mayores (Pp. 61 a 62$)$

Bustamante Pablo (2009) La interactividad como herramienta repotencializadora de los museos. Caso: Museo Argentino de Ciencias Naturales (Pp. 62 a 64)

Jiménez Álvaro Xavier (2009) El diseñador gráfico como gestor de comunicación en organizaciones sociales (ONGs) (Pp. 64 a 66)

Palma Soto Alejandro (2009) Diseño de un Weblog, como canal de comunicación directa y participativa para la señal internacional de Televisión Nacional de Chile, TV Chile (Pp. 67 a 68)

Bohórquez Piñeros Guillermo (2009) El diseño, componente estratégico de la plataforma de desarrollo de una nueva gestión cultural en Madrid (Pp. 68 a 69)

Herrera Ramos Claudia del Carmen (2009) Figuraciones del cuerpo femenino en el siglo XXI (Pp. 70 a 71)

Luzardo Alliey Ana Milagro (2009) Diseño de la interfaz gráfica web en función de los dispositivos móviles (Pp. 72 a 74)

Navia Jaramillo Silvia (2009) Empaque primario como estrategia de competitividad para las pymes exportadoras de América Latina. Una mirada a través de los productos alimenticios (Pp. 74 a 76)

Castillo Beltrán Paola Andrea (2009) Criterios transdiciplinares para el diseño de objetos lúdico-didácticos (Pp. 76 a 78)

Guerrero Blanco Clara (2009) El relato de marca: la deconstrucción narrativa de tres marcas de bebidas colombianas (Pp. 78 a 81) 
Castillo Beltrán Paola Andrea (2009) Criterios transdiciplinares para el diseño de objetos lúdico-didácticos (Pp. 83 a 164)

Cuaderno del Centro de Estudios de Diseño y Comunicación №31. (2010) Maestría en Gestión del Diseño y Maestría en Diseño de la Universidad de Palermo [Catálogo de Tesis. 1Edición. Ciclo 2004-2007]. Coordinación Fabiola Knop. Año XVIII, Abril 2010, Buenos Aires, Argentina. ISSN: 1668-0227. Esta publicación documenta y comunica los resultados alcanzados en el proyecto de investigación 16.1. Catálogos $1^{\circ}$ y $2^{\circ}$ Edición Ciclos 2004-2007 y 2008-2009 de la Maestría en Diseño de la Universidad de Palermo, y a continuación se detallan los autores y artículos contenidos en ella:

Egresados de la Maestría en Diseño de la Universidad de Palermo (Pp. 9 a 13) Majdalani Guadalupe (2007) Formato Resto-Bar. Presentación y aceptación en la Argentina (Pp. 15 a 15)

Albónico Marcelo (2007) Gestión de la imagen de la industria cinematográfica Argentina, según los diarios Clarín y La Nación en el período 2000-2002 (Pp. 16 a 25)

Pacheco Vera Fernanda Elizabeth (2007) La gestión de identidad como base para la creación de una marca empresarial: un camino para lograr una ventaja competitiva sostenible (Pp. 25 a 34)

Prieto Lozano Felipe Andrés (2007) No imagen (Pp. 34 a 39)

Zúñiga Tinizaray Vanessa Alexandra (2007) Aproximación a un vocabulario visual andino (Pp. 39 a 57)

Toala Veloz César Mauricio (2007) Diseño sustentable como expresión de identidad. Guía para la aplicación de diseño sustentable en la arquitectura vernácula (Pp. 58 a 67)

Di Bella, Daniela V. (2007) Contenidos programáticos para una carrera de arte tecnomedial en Argentina (Pp. 67 a 77)

Miguel Sánchez Isadora (2007) La constitución del diseño gráfico en República Dominicana (Pp. 77 a 78)

Brenes Bido Ivette Leilani (2007) Semiotropía y retórica de la imagen en el diseño publicitario dominicano (Pp. 78 a 82)

Reinhardt Nancy Viviana (2007) Infografía didáctica. Producción interdisciplinaria de infografías para la diversidad cultural (Pp. 82 a 86)

Franco Cañón Omar (2007) Cinco marcas colombianas (Pp. 86 a 88)

Tascón Bedoya Ruben Adolfo (2007) La Universidad.com. La Universidad online: un modelo web universitario (Pp. 89 a 92)

Melo Maturana Natalia Elena (2007) La iconografía religioso como un elemento de moda o diseño (Pp. 92 a 95)

D’Angelo Mariela (2007) La gramática del signo icónico (Pp. 95 a 97)

Zena Marcela Verónica (2007) Manifestaciones de la cultura promovidas desde la iniciativa pública: el caso del Gobierno de la Ciudad (Pp. 98 a 101)

Galanternik Noemi (2007) La evolución del diario impreso en la Argentina entre 1994 y 2004 (Pp. 101 a 106) 
Cofone María de los Milagros (2007) Estructuras sinérgicas de marca. Cobranding y gestión estratégica como agregado de valor a los activos de marca (Pp. 106 a 108)

Novoa Montoya Andrés Ricardo (2007) El lenguaje objeto-corporal (Pp. 108 a 117)

Reinhardt Nancy Viviana (2007) Infografía Didáctica: producción interdisciplinaria de infografías didácticas para la diversidad cultural (Pp. 119 a 191)

Catálogo de Tesis de Maestría en Gestión del Diseño (UP) Como parte de las iniciativas de la Línea se ha dado comienzo a la creación y gestión periódica del sitio online que recopila las publicaciones de los trabajos de Tesis Aprobadas.

\section{b)- Congresos / Coloquios / Plenarios}

I Coloquio de Investigación y Desarrollo en Diseño Latino. Universidad de Palermo, 26 de Octubre de 2016.

En dos comisiones que sesionaron en paralelo del Reservorio Gráfico y Digital de Tesis de Posgrado en Diseño: Investigación y Política Editorial Investigación. Metodología y Técnicas, se reunieron en Coloquio para debatir y presentar los resultados de sus investigaciones (reflexiones, conclusiones y avances) los maestrandos cuyas tesis integran el proyecto 16.1. Catálogos $1^{\circ}$ y $2^{\circ}$ Edición Ciclo 2004-2007 y Ciclo 2008-2009 (Cuaderno 31 y Cuaderno 38) de la Maestría en Diseño de la Universidad de Palermo.

A continuación se detallan las ponencias presentadas de las Comisiones coordinadas por la Directora del proyecto Fabiola Knop:

- Comisión 1

Amilton Arruda, Celso Hartkopf e Isabela Moroni

City Branding como modelo de analise para cidades contemporâneas

Valentina Ignacia Campos Guzmán

Como generar proyectos no convencionales en el campo del diseño / experiencia en aula

Ana María Cravino y Jorge Eduardo Pokropek

Releyendo a Umberto Eco

Clarisa Menteguiaga

Investigación en proyectos de diseño

Marta Nydia Molina González y Liliana Sosa

Los "yo" y la identidad colectiva: autorreferencia y morfogénesis. Una propuesta metodológica de diseño

Paola Andrea Tovar Polo

Modelo de marketing para el posicionamiento de marca de productos

Monica Moura

Design brasileiro contemporâneo: inter-relações culturais e econômicas 
Willam Bernardo Ruiz Joya

Un museo virtual proyecto inspirador para descubrir y aprender con investigación a través del diseño

Germán Alexander Ruiz Liberato

Proyectos integradores de aula

\title{
- Comisión 2
}

Enrique Bonilla Rodríguez y Sandra Amelia Martí

Etnografía aplicada al Diseño industrial

Graciela Laplagne

La investigación transdisciplinaria en el ámbito de la educación superior

Lucas Furio

Metodologia interdisciplinar de projeto em Design Social

Gustavo Damin e Fernanda Henriques

O inky design e a produção editorial: coleção de livros "Ensaios em design"

Federico Del Giorgio Solfa y María Sol Sierra

Procesos de diseño y desarrollo de nuevos productos: relación con el marketing y la ingeniería

Teresita de Jesús Serrano Arias

Una estrategia de investigación desde la comunicación gráfica para el estudio y salvaguardia de grupos étnicos en peligro de desaparecer

Rafael Salguero

Etnografía como método de investigación aplicado al Diseño Gráfico

Liuba Margarita Alberti Zurita

Investigar y crear. Protocolos de presentación

Diana Moreno Reyes

Lo extraño y lo familiar en los libros Imago

Mariana Luz Oliva y Silvia Torres Luyo

Trabajar con cientibecarias: una nueva perspectiva

\section{c)- Formación de Posgrado e Impacto curricular}

La Directora Fabiola Knop es Docente de Posgrado en Diseño de la Facultad de Diseño y Comunicación e incorpora los contenidos se su investigación a sus asignaturas en posgrado.

\begin{abstract}
Research Project 16.1 presents and highlights -from the thesis works- the ties that were woven between academic training with its absolutely heterogeneous processes, criteria and mechanisms, and the skill of each discipline, being able to validate along the way a theoretical model of cross-cultural intercultural competencies specific to each thesis. The articulation of all thesis projects, account for the extensive and qualified scientific
\end{abstract}


work proposed, at each of the research levels that work on the final result of the Project, proposes an intentional search for knowledge and solutions to problems that may be of a cultural and / or disciplinary training nature. Finally, it reveals the theoretical categories proposed in each work, aspects that make them original and even some plausible to be published academically.

Keywords: Knowledge - Culture - Design - Education - Research - Methodology - Projects - Thesis

Resumo: O Projeto de Pesquisa 16.1 apresenta e destaca -a partir dos trabalhos de tese- os laços que se teceram entre a formação acadêmica com seus processos, critérios e mecanismos absolutamente heterogêneos, e a competência de cada disciplina, podendo validar ao longo do caminho, um modelo teórico de competências interculturais transculturais específicas para cada tese. A articulação de todos os projetos de teses, dando conta do extenso e qualificado trabalho científico proposto, em cada um dos níveis de pesquisa que atuam no resultado final do Projeto, propõe uma busca intencional de conhecimentos e soluções para os problemas que porventura venham a ser. de caráter formativo cultural e / ou disciplinar. Por fim, apresenta as categorias teóricas propostas em cada trabalho, aspectos que os tornam originais e até mesmo alguns que são plausíveis de serem publicados academicamente.

Palavras chave: Conhecimento - Cultura - Design - Educação - Pesquisa - Metodologia - Projetos - Tese

[Las traducciones de los resúmenes fueron realizadas a través de traductor automático] 